\title{
The Effect of Bergamot-Derived Polyphenolic Fraction on LDL Small Dense Particles and Non Alcoholic Fatty Liver Disease in Patients with Metabolic Syndrome
}

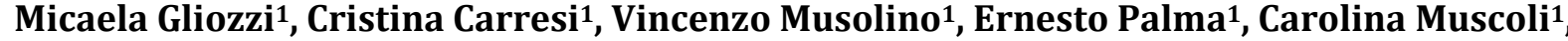 \\ Cristiana Vitale ${ }^{2}$, Santo Gratteri ${ }^{1}$, Giuseppe Muscianisi ${ }^{1}$, Elzbieta Janda ${ }^{1}$, Saverio Muscoli ${ }^{3}$, \\ Francesco Romeo ${ }^{3}$, Salvatore Ragusa1, Rocco Mollace', Ross Walker ${ }^{1}$, James Ehrlich1, \\ Vincenzo Mollace ${ }^{1^{*}}$ \\ ${ }^{1}$ Interregional Research Centre for Food Safety \& Health, University “Magna Graecia” of Catanzaro, Catanzaro, \\ Italy \\ ${ }^{2}$ San Raffaele IRCCS, Rome, Italy \\ "University of Rome "Tor Vergata", Rome, Italy \\ Email: "mollace@libero.it
}

Received 16 February 2014; revised 19 March 2014; accepted 27 March 2014

Copyright $@ 2014$ by authors and Scientific Research Publishing Inc.

This work is licensed under the Creative Commons Attribution International License (CC BY).

http://creativecommons.org/licenses/by/4.0/

c) (i) Open Access

\section{Abstract}

The occurrence of Metabolic Syndrome (MS) represents an independent risk factor for developing cardiovascular disease states in patients suffering from type 2 diabetes mellitus. Moreover, both the size of LDL particles and liver dysfunction identified as non alcoholic fatty liver disease (NAFLD) represent important biomarkers for the development of cardiometabolic risk in patients with MS. Here we studied the effect of bergamot polyphenolic fraction (BPF) in patients with MS and NAFLD. 107 patients were enrolled at the San Raffaele IRCCS (Rome). All of them showed ultrasonografic evidences of NAFLD and at least three out of five previous identified criteria for the diagnosis of MS. Patients were divided into two groups: one receiving placebo and the second receiving BPF $650 \mathrm{mg}$ twice a day for 120 consecutive days. In the group receiving BPF $650 \mathrm{mg}$ twice a day, a significant reduction of fasting plasma glucose, serum LDL cholesterol and triglycerides alongside with an increase of HDL cholesterol was found. This effect was accompanied by significant reduction of both ultrasonographic and metabolic biomarkers of NAFLD. Moreover, a significant reduction of small dense LDL particles, as detected via proton NMR Spectroscopy, was found

\footnotetext{
${ }^{*}$ Corresponding author.
}

How to cite this paper: Gliozzi, M., et al. (2014) The Effect of Bergamot-Derived Polyphenolic Fraction on LDL Small Dense Particles and Non Alcoholic Fatty Liver Disease in Patients with Metabolic Syndrome. Advances in Biological Chemistry, 4, 129-137. http://dx.doi.org/10.4236/abc.2014.42017 
after BPF treatment. In conclusion, our data confirm the beneficial effect of bergamot-extract in patients with MS an effect highlighted by significant reduction of small dense LDL particles and by improvement of NAFLD biomarkers. This suggests a potential preventive role of bergamot derivatives in reducing cardiometabolic risk.

\section{Keywords}

\section{Metabolic Syndrome; Non Alcoholic Fatty Liver Disease; Cardiometabolic Risk; Bergamot Polyphenolic Fraction}

\section{Introduction}

Evidence has been collected showing that the occurrence of metabolic syndrome (MS) is associated to increased risk of developing cardiovascular diseases and type 2 diabetes [1]. In particular, MS has been found to double the cardiometabolic risk and to enhance the risk of developing type 2 diabetes by five times [2].

Besides imbalanced glycemic control has been associated with parameters of vascular damage [3], increasing hyperglycemia in type 2 diabetes does not contribute to the cardiovascular risk to the same extent as it does in type 1 diabetes [4], pointing to the importance of nonglycemic related risk factors belonging to the MS. Numerous studies demonstrated an increased cardiovascular risk in patients with MS prior to the development of overt hyperglycemia [5] [6]. Likewise, patients with type 2 diabetes or MS have an increased cardiovascular risk despite optimal control of other risk factors as low-density lipoprotein cholesterol (LDL-C) [7].

In the context of the shortcomings of commonly assessed risk factors in individuals with features of the MS, the characterization and sub-classification of LDL and high-density lipoprotein HDL particles emerged as a tool that may offer a better risk prediction. A decrease of HDL associated with an increase in small, dense LDL particles (sdLDL), class III and IV, is closely associated with an increased cardiovascular risk, independently of the traditional risk factors both in patients with [8]-[10] and without [11]-[13] diabetes or MS. Individual LDL particle profiles normally cluster into two patterns of LDL size distribution: the majority of profiles demonstrates a predominance of large or medium sized LDL particles (LDL pattern A), whereas a substantial minority exhibits the LDL pattern B with a higher proportion of smaller LDL particles [14].

Several aspects must be taken into account when evaluating the potential role of sdLDL in patients with MS. In particular, evidence has been accumulated suggesting that the formation of sdLDL particles seem to be favoured in the presence of insulin resistance and elevated triglicerydes [15] [16]. Although the mechanism underlying this effect is still unknown, it is likely also that such a condition involves an increased hepatic uptake of LDL particles, possibly due to the upregulation of hepatic LDL receptor activity and/or increased clearance of LDL particles due to their altered composition and liver dysfunction.

Recently, it has been shown that MS is associated with non alcoholic fatty liver disease state (NAFLD). In particular, NAFLD is one of the most common causes of liver-related mortality worldwide [17]. NAFLD encompasses a morphological spectrum from simple fatty liver (SFL), non-alcoholic steatohepatitis (NASH) to hepatic cirrhosis [18]. NAFLD has been identified as the hepatic manifestation of MS [19] and the association between NAFLD and MS has been reported in earlier observational studies [20]-[22]. There are a number of common mechanisms underlying the development of NAFLD and MS. For example, they may have the same pathophysiological basis of insulin resistance [23]. A systematic review suggested a potential predictive effect of liver fat on the presence of MS [23]. However, no evidence was provided regarding the causal association between NAFLD and MS. Moreover, many compounds used for counteracting the detrimental effect of MS on cardiovascular system, such as statins, have not been found to produce beneficial effect on liver dysfunction or enhanced fat accumulation in the liver [24], as well as asymptomatic elevation of serum alanine (ALT) and aspartate amino-transferases (AST) [25].

Recently, we found that bergamot-derived polyphenolic fraction (BPF) produces significant reduction of serum cholesterol, triglycerides and fasting glucose in hyperlipemic patients [26] [27], an effect which occurs at multilevels including an enhanced metabolism of lipoproteins at the hepatic level [28]. In addition, the administration of bergamot juice in rats fed with a hypercholesterolemic diet, significantly reduces liver steatosis, 
though the mechanism is still unclear [26].

The present experiments have been performed to study the effect of BPF on lipoprotein subfraction profile and NAFLD in patients with MS.

\section{Materials and Methods}

\subsection{Study Design}

The study has been carried out in 107 patients suffering from MS and NAFLD enrolled at the San Raffaele IRCCS, Rome and at the Ambulatory of alimentary toxicology at the University of Catanzaro, Italy. Diagnosis of MS was based, according to the NCEP-ATP III criteria, on the occurrence of at least three out of five components of the syndrome, such as: elevated fasting plasma glucose (or previously diagnosed type 2 diabetes), abdominal obesity measured by waist circumference, elevated triglycerides, decreased HDL-cholesterol and increased arterial blood pressure. The occurrence of concomitant NAFLD was detected by ultrasonographic examination of patients. Only patients with a hepato-renal index (see belove) in the range of 2.5 - 3.5 were admitted to the study. After randomization, patients were taking BPF $650 \mathrm{mg}$ twice a day before meals for 120 consecutive days.

All participants provided written informed consent prior to participation. The study was approved by the local Medical Ethics Committee.

\subsection{Plant Material}

C. bergamia Risso \& Poiteau fruits were collected from plants located in a range of $90 \mathrm{Km}$ from Bianco to Reggio Calabria, Italy.

\subsection{Preparation of BPF}

Bergamot juice was obtained from peeled-off fruits by squeezing. The juice was oil fraction-depleted by stripping, clarified by ultra-filtration and loaded on to a suitable polystyrene resin column able to absorb polyphenol compounds of molecular weight between 300 to 600 Da (Mitsubishi). Polyphenol fractions were eluted by a $1 \mathrm{mM} \mathrm{KOH}$ solution. The basic eluate was incubated at a rocking platform to reduce the furocumarin content. The shaking time was adjusted proportionally to the amount of furocumarin contaminants. Next, the phytocomplex derived from the process performed to remove furocumarins was neutralized by filtration on cationic resin at acidic $\mathrm{pH}$. Finally it was vacuum dried and minced to the desired particle size to obtain BPF powder. BPF powder was analysed for flavonoid, furocumarin and other polyphenol content which was standardized at $38 \%$. In addition, all toxicological analyses were performed, including heavy metal, pesticide, phthalate and sinephrine content which revealed the absence of known toxic compounds at significant levels (data not shown). Standard microbiological test showed the final BPF was free of mycotoxins and contaminating bacteria.

Finally, $650 \mathrm{mg}$ aliquots of the BPF powder were encapsulated together with 100 mg oleuropein $20 \%$ and 50 $\mathrm{mg}$ of ascorbic acid into suitable gelatin capsules by a semi-automatic gelatin encapsulation device employing an authorized pharmaceutical manufacturer (Plants, Messina, Italy). All procedures have been performed according to Good Manufacture Practice (GMP) headlines of European Legislation.

The main flavonoids identified in 38\% BPF were neoeriocitrin (370 ppm), naringin (520 ppm), and neohesperidin (310 ppm). Tablets containing $1000 \mathrm{mg}$ of maltodestrin supplemented with $50 \mathrm{mg}$ ascorbic acid were used as placebo.

\subsection{Data Collection and Measurements}

At baseline, all participants were asked to come in the morning after fasting for $>10 \mathrm{~h}$. Fasting blood samples were collected for measuring conventional risk factors of liver or cardiovascular disease (CVD), including lipids, glucose, transaminases and inflammatory markers. Face-to-face interviews and physical examinations were performed by well-trained nurses or physicians. Demographic and lifestyle information was collected by a standardized questionnaire. Alcohol consumption was classified into never, occasional, moderate and excessive based on the usual frequency of intake and the usual amount per occasion. Those who did not drink any alcohol throughout their life were classified as non-drinkers. Those who drank $<1$ occasion per week or drank only on 
special occasions in the past one year were classified as occasional drinkers. Moderate drinkers were regular drinkers ( $\geq 1$ occasion per week) who drank $<20 \mathrm{~g}$ alcohol per day in males or $<10 \mathrm{~g}$ alcohol per day in females, while excessive drinkers with alcohol consumption of $\geq 20$ g per day in males or $\geq 10$ g per day in females were not included in this study.

\subsection{Diagnostic Criteria of NAFLD}

Following exclusion of subjects with excessive alcohol consumption and viral or autoimmune liver disease, NAFLD was diagnosed by abdominal ultrasound, which is a widely accessible imaging technique with high diagnostic accuracy and reliability for the detection of fatty liver. An ultrasonographic examination was performed by an experienced radiologist using a real-time scanner (3.5 MHz; Mod. Aplio, Toshiba, Japan) equipped with a convex-array probe. All subjects were evaluated in the left lateral recumbent position of $15^{\circ}-20^{\circ}$ to see the liver parenchyma and the right kidney cortex was seen contemporaneously.

The brightness of both zones was examined. The liver was recorded from the intercostal space, posing the region of interest (of $1.5 \mathrm{~cm} \times 1.5 \mathrm{~cm}$ ) in the mid or anterior axillary line (seventh or eighth intercostal space).

The right kidney was evaluated, posing the region of interest $(0.5 \mathrm{~cm} \times 0.5 \mathrm{~cm})$ in the cortical zone. HepatoRenal Index Difference (HRI-diff: Echo Levels in the Liver-Echo Levels in the Kidney) was estimated using built-in software on the scanner enabling local measurement of attenuation in dB. Mild steatosis was diagnosed for hyperechogenic liver tissue (compared with the kidney cortex) when the sonographic index results were between 1 and 2. Values between 2 and 2.5 were indicative of moderate liver steatosis. Finally, hepatic steatosis was judged as severe when the hepatorenal ratio was $>2.5$. In each case, the calculation of the hepatorenal index was repeated at least twice.

\subsection{Laboratory Measurements}

Plasma samples were collected in EDTA-containing vials after a $12 \mathrm{~h}$ overnight fast on day 0 and after 120 days of treatment with BPF (650 mg twice a day before each meal).

Total cholesterol (in mg/dL), high density lipoprotein cholesterol (HDL-C), LDL-C, triglycerides (TG) and fasting plasma glucose, steato test, ALT, AST and $\gamma$-glutamyl peptidase ( $\gamma$-GT) were evaluated at baseline and after four months of treatment with BPF. Under the same treatment schedule with BPF, lipoprotein particles were detected by means of proton NMR spectroscopy technique which simultaneously measures the particle concentrations of lipoprotein subclasses of different sizes. Each of the lipoprotein subclasses emits a distinctive NMR signal, the amplitude of which is directly proportional to the number of subclass particles emitting the signal. Importantly, variation in lipoprotein particle lipid composition does not alter the relationship between the NMR signal and the particle size. The NMR also provides calculated values for mean very-low-density lipoprotein (VLDL), LDL, and HDL particle sizes plus estimates of total and VLDL, TG and HDL cholesterol. NMRbased estimates of TG and HDL cholesterol were calculated using conversion factors that assume normal lipid content of the various subclasses.

Circulating TNF- $\alpha$ levels was measured by ELISA using kits from R \& D Systems and Biosource and, finally, C-reactive protein (CRP) was measured using nephelometry and reagents from Beckman Coulter.

\subsection{Statistical Analysis}

For continuous variables, baseline differences between the BPF and baseline levels were assessed using Student's t test for independent samples. Data analyses were conducted using SPSS software (version 18.0).

\section{Results}

Demographics and glyco-lipemic serum profile in patients with MS and NAFLD at baseline as well as following 120 consecutive days of treatment with BPF (650 mg given orally twice a day before meals) are shown in Table 1. Data on biomarkers of hepatic function, steato test and inflammatory biomarkers such as TNF-a and CRP are also displayed.

Basal levels showed an elevated BMI combined with a mixed hyperlipemia (elevated total cholesterol plus hypertriglyceridemia). High LDL cholesterol was associated with reduced HDL cholesterol and elevated fasting serum glucose, suggesting the occurrence of MS. The elevated lipemic and glycemic biomarkers were accompa- 
nied by changes in transaminases and steato test, suggesting that MS was accompanied by NAFLD. Moreover, inflammatory biomarkers were found elevated in patients at baseline thus suggesting that MS combined with hepatic dysfunction was accompanied by an inflammatory state.

In patients treated with BPF (650 mg given orally twice a day before meals) for 120 consecutive days a significant reduction of serum total cholesterol, LDL-C and triglycerides was found (Table 1). This effect was accompanied by significant reduction of serum glucose, transaminases, gamma-glutamyl-transferase, steato test and inflammatory biomarkers such as TNF-a and CRP.

Moreover, a substantial re-arrangement of lipoprotein particles was found compared to the baseline profile (Table 2). Indeed, as measured using NMR, data detected in patients following 120 days of BPF treatment showed relevant changes in mean particle diameters for VLDL, LDL, and HDL ( $P<0.05$ for all) compared to the baseline levels (Table 2).

In particular, BPF was found able to decrease the mean concentration of IDL particles by $51 \%$, to increase large LDL by 38\%, and to decrease small LDL by 35\%. Moreover, 120 day treatment with BPF lead to $20 \%$ increase of total HDL particles, mainly due to the increase of large HDL ( $\mathrm{P}=0.05$ vs baseline levels).

The beneficial effect of treating patients with MS associated with NAFLD with BPF was confirmed by data obtained when studying ultrasonografic pattern of NAFLD. Indeed, hepatorenal index was significantly $(\mathrm{P}<$ 0.05 ) reduced from $2.8 \pm 0.4$ to $1.5 \pm 0.5$ by treatment with BPF showing a reduction of liver brightness. This suggests that treating patients suffering from mild to severe NAFLD associated with MS with BPF (650 mg twice a day for 120 consecutive days) leads to reduction of hepatic ultrosonographic pattern of steatosis.

No side effects related to the treatment with BPF have been described overall the study period, thus confirming data on safety profile of bergamot extract previously shown by our and other groups.

\section{Discussion}

The present data confirmed previous results showing that BPF, the extract of bergamot juice rich in polyphenols,

Table 1. Demographic, haematological and ultrasonic data obtained at baseline and after treatment with BPF (650 mg twice a day for 120 consecutive days) in 107 patients suffering from metabolic syndrome (MS) and Non-Alcoholic Fatty Liver Disease (NAFLD). (Data are expressed as mean \pm SD for each value; a $\mathrm{P}$ value of $<0.05$ between values at baseline and after BPF treatment was taken as significant ${ }^{*}$ ).

\begin{tabular}{ccc}
\hline Hematological, inflammatory and ultrasonic biomarkers & Baseline & BPF \\
\hline Patients undergoing BPF (650 $\times$ 2 daily) for 120 consecutive days & 107 & \\
Age (yr) & $56 \pm 12$ & \\
Sex (M/F) & $64 / 43$ & \\
BMI (kg/m $\left.{ }^{2}\right)$ & $29.4 \pm 2.01$ & $28.2 \pm 1.53$ \\
Fasting plasma glucose (mg/mL) & $118 \pm 1.4$ & $98 \pm 0.8^{*}$ \\
Total Cholesterol (mg/dL) & $245 \pm 8.3$ & $182 \pm 7.1^{*}$ \\
LDL-C (mg/mL) & $162 \pm 4.3$ & $101 \pm 1.8^{*}$ \\
HDL-C (mg/mL) & $38 \pm 3.8$ & $49 \pm 4^{*}$ \\
Triglycerides (mg/mL) & $232 \pm 5.1$ & $160 \pm 4.8^{*}$ \\
Steato test & $0.74 \pm 0.12$ & $0.44 \pm 0.09^{*}$ \\
ALT (U/L) & $54 \pm 5.4$ & $36 \pm 5.3^{*}$ \\
AST (U/L) & $52 . \pm 6.4$ & $41 \pm 5.2^{*}$ \\
$\gamma$-GT (IU/L) & $38 \pm 5.2$ & $29.33 \pm 1.1^{*}$ \\
Hs-CRP (mcg/dl) & $1.2+0.8$ & $0.94+0.6^{*}$ \\
TNF- $\alpha(\mathrm{pg} / \mathrm{mL})$ & $14.4 \pm 1.9$ & $10.7 \pm 1.7^{*}$ \\
Hepatorenal index & $2.8 \pm 0.4$ & $1.5 \pm 0.5^{*}$ \\
\hline
\end{tabular}


Table 2. Plasma lipoprotein particle size and concentration in patients with MS and NAFLD at the baseline and after 120 consecutive day treatment with BPF $650 \mathrm{mg} \times 2$ daily. (Data are expressed as mean \pm SD for each value; a $\mathrm{P}$ value of $<0.05$ between values at baseline and after BPF treatment was taken as significant ${ }^{*}$ ).

\begin{tabular}{ccc}
\hline Plasma lipoprotein plasma diameter, $\boldsymbol{n m}$ & Baseline & BPF \\
VLDL & $55.3 \pm 6.4$ & $44.5 \pm 5.2^{*}$ \\
LDL & $22.6 \pm 1.7$ & $18.0 \pm 0.8^{*}$ \\
HDL & $7.5 \pm 0.8$ & $9.6 \pm 0.9^{*}$ \\
Plasma lipoprotein particles, $\mathbf{n m o l} / \mathbf{L}$ & & \\
Total VLDL & $83 \pm 14$ & $54 \pm 12^{*}$ \\
Large VLDL & $4.2 \pm 2$ & $1.8 \pm 1.3^{*}$ \\
Medium VLDL & $31 \pm 9$ & $14 \pm 8^{*}$ \\
Small VLDL & $43 \pm 9$ & $38 \pm 10$ \\
Total LDL & $1477 \pm 75$ & $1293 \pm 101^{*}$ \\
IDL & $77 \pm 16$ & $38 \pm 10^{*}$ \\
Large LDL & $424 \pm 87$ & $653 \pm 95^{*}$ \\
Small LDL & $986 \pm 105$ & $612 \pm 98^{*}$ \\
Total HDL & $30 \pm 2$ & $36 \pm 3^{*}$ \\
Large HDL & $5 \pm 3$ & $15 \pm 4^{*}$ \\
Medium HDL & $7 \pm 4$ & $7 \pm 3$ \\
Small HDL & $18 \pm 5$ & $14 \pm 4^{*}$ \\
\hline
\end{tabular}

reduces both cholesterol, triglycerides and glucose in patients suffering from MS [26] [27]. This effect is accompanied by reduction of LDL-C and elevation of HDL-C, thus suggesting a beneficial effect in the lipemic profile of patients undergoing MS. The added value and novelty of data reported in this study using BPF in such a subgroup of patients with elevated cardiometabolic risk, is also displayed by prominent re-arrangement of lipoprotein particle profile found following 120 day BPF treatment. Indeed, BPF reduced LDL small-size, atherogenic particles and enhanced large-size anti-atherogenic HDL lipoprotein particles. This effect, combined with reduction of inflammatory biomarkers, suggests that BPF leads to an attenuation of atherogenic risk in patients with MS.

The mechanism of such an effect in lipoprotein particle size is not clear to date. The combined effect of BPF in reducing both cholesterol and triglycerides may well explain lipoprotein re-arrangement due to the prolonged BPF treatment. Indeed, an increased clearance of TG-rich lipoprotein particles makes these particles became better substrates for lipoprotein lipase. This would be expected to result in decreased levels of large and medium-sized VLDL and perhaps even intermediate density lipoprotein (IDL), which contains roughly equal amounts of TG and cholesterol.

The increased cascade of VLDL to IDL to LDL would result in increased numbers of large LDL particles and provide surface constituents for the formation of large HDL. The formation of small LDL is mainly due to cholesteryl ester transfer protein-mediated exchange of VLDL-TG for LDL cholesterol ester and the subsequent hydrolysis of LDL-TG. The decrease in large and medium VLDL diminishes the cholesteryl ester transfer protein-mediated exchange, decreasing the formation and number of small LDL particles.

The improvement of hepatocyte functionality found in patients with MS and associated NAFLD after taking BPF might also contribute in the amelioration in lipoprotein profile thereby attenuating cardiometabolic risk.

Previous studies demonstrated that insulin resistance almost universally induces NAFLD [29] [30]. It is known that this condition may precede the development of cardiovascular disease [31] [32]. To confirm the connection between NAFLD and atherosclerosis, carotid atherosclerosis has recently been detected in patients with NAFLD [33]. Pathophysiological mechanisms responsible for that effect include an increased lipolysis and increased delivery of free fatty acids to the liver [34]. Other abnormalities that can contribute to fat accumula- 
tion in the liver include decreased synthesis of apolipoproteins and microsomal transfer protein gene polymorphism, both conditions that lead to decreased export of triglycerides out of the liver [35]. The improvement of steato test and hepatorenal index in patients with MS and NAFLD following BPF treatment gives a quantitative estimation of steatosis and leads to the conclusion that BPF improves both liver function and signs of chronic liver inflammation, as confirmed by reduction of TNF-a and CRP. Mild to moderate elevation of serum aminotransferases (ALT and AST) found in our subjects at baseline represents the most common abnormality found in patients with NAFLD. Their serum levels were significantly reduced after BPF, thereby confirming data obtained with steato test and hepatorenal index. The mechanism of hepato-protective effect of BPF still remains to be elucidated.

Previous studies showed that both bergamot juice and BPF act as a cytoprotectant agents in the liver of rats undergoing hypercolesterolemic diet [28] [36]. This should be due to BPF activities in oxidative inflammation and changes in hepatocyte membrane permeability probably via stabilization of the hepatocyte membrane structure, thereby preventing toxins from entering the cells. In addition, it has recently been shown that non-nutritive constituents of Citrus family fruits, such as pectin and flavonoids found in peel extracts, cause lowering of serum and/or tissue cholesterol levels by modulating hepatic 3-hydroxy-3-methylglutaryl coenzyme A (HMGCoA) levels, possibly by binding bile acids and increasing the turnover rate of blood and liver cholesterol [37]-[41]. Since BPF showed to be able to enhance the excretion of fecal sterols in rats [42], such a mechanism may contribute to both the hypolipidemic and hepato-protective properties of bergamot derivatives. Moreover, a major contribution to the hypolipidemic response found in patients undergoing BPF treatment seems to be related to the modulatory properties of the flavonone glycoside component of BPF, naringin and neo-hesperidin in particular. Indeed, evidence exists that dietary hesperetin reduces hepatic TG accumulation associated with a reduced activity of TG synthetic enzyme, PAP [43]. Thus, BPF polyphenolic components, via multi-action properties, reduce liver accumulation of fat thereby producing an overall improvement of liver function.

\section{Conclusion}

In conclusion, our data show that bergamot-deriving polyphenolic fraction given in patients with MS and NAFLD, leads to concomitant amelioration of the lipemic and glycemic serum profile and to substantial reduction of liver steatosis. This effect, alongside with a reduction of pro-atherogenic small dense LDL and enhancement of anti-atherogenic high dense HDL, shed new light on the potential use of bergamot-extract for reducing cardiometabolic risk in patients with MS.

\section{Acknowledgements}

This work was supported by PON-MIUR 00359. We would like to thank Mr. Giovanni Politi for skillful technical assistance, Dr. Giuseppe Pittari for statistical analysis and Malara Group for preparing bergamot extract.

\section{References}

[1] Haffner, S.M., Lehto, S., Rönnemaa, T., Pyörälä, K. and Laakso, M. (1998) Mortality from Coronary Heart Disease in Subjects with Type 2 Diabetes and in Non Diabetic Subjects with and without Prior Myocardial Infarction. New England Journal of Medicine, 339, 229-234. http://dx.doi.org/10.1056/NEJM199807233390404

[2] Thalhammer, C., Balzuweit, B., Busjahn, A., Walter, C., Luft, F.C. and Haller, H. (1999) Endothelial Cell Dysfunction and Arterial Wall Hypertrophy Are Associated with Disturbed Carbohydrate Metabolism in Patients at Risk for Cardiovascular Disease. Arteriosclerosis, Thrombosis, and Vascular Biology, 19, 1173-1179. http://dx.doi.org/10.1161/01.ATV.19.5.1173

[3] Juutilainen, A., Lehto, S., Rönnemaa, T., Pyörälä, K. and Laakso, M. (2008) Similarity of the Impact of Type 1 and Type 2 Diabetes on Cardiovascular Mortality in Middle-Aged Subjects. Diabetes Care, 31, 714-719. http://dx.doi.org/10.2337/dc07-2124

[4] Hu, F.B., Stampfer, M.J., Haffner, S.M., Solomon, C.G., Willett, W.C. and Manson, J.E. (2002) Elevated Risk of Cardiovascular Disease Prior to Clinical Diagnosis of Type 2 Diabetes. Diabetes Care, 25, 1129-1134. http://dx.doi.org/10.2337/diacare.25.7.1129

[5] Isomaa, B., Almgren, P., Tuomi, T., Forsén, B., Lahti, K., Nissén, M., Taskinen, M.R. and Groop, L. (2001) Cardiovascular Morbidity and Mortality Associated with the Metabolic Syndrome. Diabetes Care, 24, 683-689. http://dx.doi.org/10.2337/diacare.24.4.683 
[6] Sampson, U.K., Fazio, S. and Linton, M.F. (2012) Residual Cardiovascular Risk Despite Optimal LDL Cholesterol Reduction with Statins: The Evidence, Etiology, and Therapeutic Challenges. Current Atherosclerosis Reports, 14, 110. http://dx.doi.org/10.1007/s11883-011-0219-7

[7] Berneis, K., Jeanneret, C., Muser, J., Felix, B. and Miserez, A.R. (2005) Low Density Lipoprotein Size and Subclasses Are Markers of Clinically Apparent and Non-Apparent Atherosclerosis in Type 2 Diabetes. Metabolism, 54, $227-234$.

[8] Krauss, R.M. (2004) Lipids and Lipoproteins in Patients with Type 2 Diabetes. Diabetes Care, 27, 1496-1504. http://dx.doi.org/10.2337/diacare.27.6.1496

[9] Rizzo, M., Pernice, V., Frasheri, A., Di Lorenzo, G., Rini, G.B., Spinas, G.A. and Berneis, K. (2009) Small, Dense Low-Density Lipoproteins (LDL) Are Predictors of Cardio and Cerebro-Vascular Events in Subjects with the Metabolic Syndrome. Clinical Endocrinology (Oxf), 70, 870-875. http://dx.doi.org/10.1111/j.1365-2265.2008.03407.x

[10] Austin, M.A., Breslow, J.L., Hennekens, C.H., Buring, J.E., Willett, W.C. and Krauss, R.M. (1988) Low-Density Lipoprotein Subclass Patterns and Risk of Myocardial Infarction. JAMA, 260, 1917-1921. http://dx.doi.org/10.1001/jama.1988.03410130125037

[11] Feingold, K.R., Grunfeld, C., Pang, M., Doerrler, W. and Krauss, R.M. (1992) LDL Subclass Phenotypes and Triglyceride Metabolism in Non-Insulin Dependent Diabetes. Arteriosclerosis, Thrombosis, and Vascular Biology, 12, 14961502. http://dx.doi.org/10.1161/01.ATV.12.12.1496

[12] Krayenbuehl, P.A., Wiesli, P., Schmid, C., Lehmann, R., Spinas, G.A. and Berneis, K. (2008) Insulin Sensitivity in Type 2 Diabetes Is Closely Associated with LDL Particle Size. Swiss Medical Weekly, 138, 275-280.

[13] Chait, A., Brazg, R.L., Tribble, D.L. and Krauss, R.M. (1993) Susceptibility of Small, Dense, Low-Density Lipoproteins to Oxidative Modification in Subjects with the Atherogenic Lipoprotein Phenotype, Pattern B. American Journal of Medicine, 94, 350-356. http://dx.doi.org/10.1016/0002-9343(93)90144-E

[14] Younis, N., Charlton-Menys, V., Sharma, R., Soran, H. and Durrington, P.N. (2009) Glycation of LDL in Non-Diabetic People: Small Dense LDL Is Preferentially Glycated Both in Vivo and in Vitro. Atherosclerosis, 202, 162-168. http://dx.doi.org/10.1016/j.atherosclerosis.2008.04.036

[15] American Diabetes Association. Position Statement (2012) American Diabetes Association Standards of Medical Care in Diabetes. Diabetes Care, 35, S11-S63.

[16] Eknoyan, G., Hostetter, T., Bakris, G.L., Hebert, L., Levey, A.S., Parving, H.H., Steffes, M.W. and Toto, R. (2003) Proteinuria and Other Markers of Chronic Kidney Disease: A Position Statement of the National Kidney Foundation (NKF) and the National Institute of Diabetes and Digestive and Kidney Diseases (NIDDK). American Journal of Kidney Diseases, 42, 617-622. http://dx.doi.org/10.1016/S0272-6386(03)00826-6

[17] Ong, J.P., Pitts, A. and Younossi, Z.M. (2008) Increased Overall Mortality and Liver-Related Mortality in Non-Alcoholic Fatty Liver Disease. Journal of Hepatology, 49, 608-612. http://dx.doi.org/10.1016/j.jhep.2008.06.018

[18] Wang, Y., Li, Y.Y., Nie, Y.Q., Zhou, Y.J., Cao, C.Y. and Xu, L. (2013) Association between Metabolic Syndrome and the Development of Non-Alcoholic Fatty Liver Disease. Experimental and Therapeutic Medicine, 6, 77-84.

[19] Tarantino, G. (2007) Should Non Alcoholic Fatty Liver Disease Be Regarded as a Hepatic Illness Only? World Journal of Gastroenterology, 13, 4669-4672.

[20] Hamaguchi, M., Kojima, T., Takeda, N., Nakagawa, T., Taniguchi, H., Fujii, K., Omatsu, T., Nakajima, T., Sarui, H., Shimazaki, M., Kato, T., Okuda, J. and Ida, K. (2005) The Metabolic Syndrome as a Predictor of Nonalcoholic Fatty Liver Disease. Annals of Internal Medicine, 143, 722-728. http://dx.doi.org/10.7326/0003-4819-143-10-200511150-00009

[21] Kim, H.M., Kim, D.J., Jung, I.H., Park, C. and Park, J. (2007) Prevalence of the Metabolic Syndrome among Korean Adults Using the New International Diabetes Federation Definition and the New Abdominal Obesity Criteria for the Korean People. Diabetes Research and Clinical Practice, 77, 99-106. http://dx.doi.org/10.1016/j.diabres.2006.10.009

[22] Seo, H.I., Cho, Y.K., Lee, W.Y., Rhee, E.J., Sung, K.C., Kim, B.S., Son, B.H., Shin, J.H., Joo, K.J., Hong, H.P., Chae, S.W. and Jin, W. (2012) Which Metabolic Syndrome Criteria Best Predict the Presence of Non-Alcoholic Fatty Liver Disease? Diabetes Research and Clinical Practice, 95, 19-24. http://dx.doi.org/10.1016/j.diabres.2011.08.013

[23] Kotronen, A. and Yki-Järvinen, H. (2008) Fatty Liver: A Novel Component of the Metabolic Syndrome. Arteriosclerosis, Thrombosis, and Vascular Biology, 28, 27-23. http://dx.doi.org/10.1161/ATVBAHA.107.147538

[24] Aguirre, L., Hijona, E., Macarulla, M.T., Gracia, A., Larrechi. I., Bujanda. L., Hijona, L. and Portillo, M.P. (2013) Several Statins Increase Body and Liver Fat Accumulation in a Model of Metabolic Syndrome. Journal of Physiology and Pharmacology, 64, 281-288.

[25] Tsai, C.H., Li, T.C. and Lin, C.C. (2008) Metabolic Syndrome as a Risk Factor for Non-Alcoholic Fatty Liver Disease. Southern Medical Journal, 101, 900-905. http://dx.doi.org/10.1097/SMJ.0b013e31817e8af9

[26] Mollace, V., Sacco, I., Janda, E., Malara, C., Ventrice, D., Colica, C., Visalli, V., Muscoli, S., Ragusa, S., Muscoli, C., 
Rotiroti, D. and Romeo, F. (2011) Hypolipemic and Hypoglycaemic Activity of Bergamot Polyphenols: From Animal Models to Human Studies. Fitoterapia, 82, 309-316. http://dx.doi.org/10.1016/j.fitote.2010.10.014

[27] Gliozzi, M., Walker, R., Muscoli, S., Vitale, C., Gratteri, S., Carresi, C., Musolino, V., Russo, V., Janda, E., Ragusa, S., Aloe, A., Palma, E., Muscoli, C., Romeo, F. and Mollace, V. (2013) Bergamot Polyphenolic Fraction Enhances Rosuvastatin-Induced Effect on LDL-Cholesterol, LOX-1 Expression and Protein Kinase B Phosphorylation in Patients with Hyperlipidemia. International Journal of Cardiology, 170, 140-145. http://dx.doi.org/10.1016/j.ijcard.2013.08.125

[28] Janda, E., Parafati, M., Aprigliano, S., Carresi, C., Visalli, V., Sacco, I., Ventrice, D., Mega, T., Vadalá, N., Rinaldi, S., Musolino, V., Palma, E., Gratteri, S., Rotiroti, D. and Mollace, V. (2013) The Antidote Effect of Quinone Oxidoreductase 2 Inhibitor against Paraquat-Induced Toxicity in Vitro and in Vivo. British Journal of Pharmacology, 168, $46-59$. http://dx.doi.org/10.1111/j.1476-5381.2012.01870.x

[29] Sanyal, A.J. (2002) AGA Technical Review on Nonalcoholic Fatty Liver Disease. Gastroenterology, 123, $1705-1725$. http://dx.doi.org/10.1053/gast.2002.36572

[30] Brea, A., Mosquera, D., Martín, E., Arizti, A., Cordero, J.L. and Ros, E. (2005) Nonalcoholic Fatty Liver Disease Is Associated with Carotid Atherosclerosis: A Case-Control Study. Arteriosclerosis, Thrombosis, and Vascular Biology, 25, 1045-1050. http://dx.doi.org/10.1161/01.ATV.0000160613.57985.18

[31] Marchesini, G., Brizi, M., Morselli-Labate, A.M., Bianchi, G., Bugianesi, E., McCullough, A.J., Forlani, G. and Melchionda, N. (1999) Association of Nonalcoholic Fatty Liver Disease with Insulin Resistance. American Journal of Medicine, 107, 450-455. http://dx.doi.org/10.1016/S0002-9343(99)00271-5

[32] Hamaguchi, M., Kojima, T., Takeda, N., Nagata, C., Takeda, J., Sarui, H., Kawahito, Y., Yoshida, N., Suetsugu, A., Kato, T., Okuda, J., Ida, K. and Yoshikawa, T. (2007) Nonalcoholic Fatty Liver Disease Is a Novel Predictor of Cardiovascular Disease. World Journal of Gastroenterology, 13, 1579-1584.

[33] Chitturi, S., Abeygunasekera, S., Farrell, G.C., Holmes-Walker, J., Hui, J.M., Fung, C., Karim, R., Lin, R., Samarasinghe, D., Liddle, C., Weltman, M. and George, J. (2002) NASH and Insulin Resistance: Insulin Hypersecretion and Specific Association with the Insulin Resistance Syndrome. Hepatology, 35, 373-379. http://dx.doi.org/10.1053/jhep.2002.30692

[34] Choudhury, J. and Sanyal, A.J. (2004) Insulin Resistance and the Pathogenesis of Nonalcoholic Fatty Liver Disease. Clinical Liver Disease, 8, 575-594. http://dx.doi.org/10.1016/j.cld.2004.04.006

[35] Bernard, S., Touzet, S., Personne, I., Lapras, V., Bondon, P.J., Berthezène, F. and Moulin, P. (2000) Association between Microsomal Triglyceride Transfer Protein Gene Polymorphism and the Biological Features of Liver Steatosis in Patients with Type II Diabetes. Diabetologia, 43, 995-999. http://dx.doi.org/10.1007/s001250051481

[36] Miceli, N., Mondello, M.R., Monforte, M.T., Sdrafkakis, V., Dugo, P., Crupi, M.L., Taviano, M.F., De Pasquale, R. and Trovato, A. (2007) Hypolipidemic Effects of Citrus bergamia Risso et Poiteau Juice in Rats Fed a Hypercholesterolemic Diet. Journal of Agricultural and Food Chemistry, 55, 10671-10677. http://dx.doi.org/10.1021/jf071772i

[37] Bok, S.H., Lee, S.H., Park, Y.B., Bae, K.H., Son, K.H., Jeong, T.S. and Choi, M.S. (1999) Plasma and Hepatic Cholesterol and Hepatic Activities of 3-Hydroxy-3-Methyl-Glutaryl-CoA Reductase and Acyl CoA: Cholesterol Transferase Are Lower in Rats Fed Citrus Peel Extract or a Mixture of Citrus Bioflavonoids. Journal of Nutrition, 129, 1182-1185.

[38] Marounek, M., Volek, Z., Synytsya, A. and Copikova, J. (2007) Effect of Pectin and Amidated Pectin on Cholesterol Homeostasis and Cecal Metabolism in Rats Fed a High-Cholesterol Diet. Physiological Research, 56, 433-442.

[39] Terpstra, A.H., Lapre, J.A., de Vries, H.T. and Beynen, A.C. (1998) Dietary Pectin with High Viscosity Lowers Plasma and Liver Cholesterol Concentration and Plasma Cholesteryl Ester Transfer Protein Activity in Hamsters. Journal of Nutrition, 128, 1944-1949.

[40] Anonymous (2002) The Hypocholesterolemic Effect of Lemon Peels, Lemon Pectin, and the Waste Stream Material of Lemon Peels in Hybrid F1B Hamsters. European Journal of Nutrition, 41, 19-26. http://dx.doi.org/10.1007/s003940200002

[41] Garcia-Diez, F., Garcia-Mediavilla, V., Bayon, J.E. and Gonzalez-Gallego, J. (1996) Pectin Feeding Influences Fecal Bile Acid Excretion, Hepatic Bile Acid and Cholesterol Synthesis and Serum Cholesterol in Rats. Journal of Nutrition, 126, 1766-1771.

[42] Pappu, A.S. and Illingworth, D.R. (1989) Contrasting Effects of Lovastatin and Cholestyramine on Low-Density Lipoprotein Cholesterol and 24-Hour Urinary Mevalonate Excretion in Patients with Heterozygous Familial Hypercholesterolemia. Journal of Laboratory and Clinical Medicine, 114, 554-562.

[43] Cha, J.Y., Cho, Y.S., Kim, I., Anno, T., Rahman, S.M. and Yanagita, T. (2001) Effect of Hesperetin, a Citrus Flavonoid, on the Liver Triacylglycerol Content and Phosphatidate Phosphohydrolase Activity in Orotic Acid-Fed Rats. Plant Foods for Human Nutrition, 56, 349-358. http://dx.doi.org/10.1023/A:1011884200848 